\title{
"Influencia del Nivel de Calidad Institucional en el Crecimiento Económico del Perú / 2006-2010"
}

\author{
"Influence of Level of Institutional Quality on Economic Growth \\ Perú/2006-2010"
}

\author{
${ }^{1}$ Jesús Amadeo Olivera Cáceres
}

\begin{abstract}
RESUMEN:
En cuanto a materiales y métodos; el trabajo corresponde a una investigación de tipo documental; cuyo nivel corresponde al relacional. La información se obtuvo a través de la aplicación del instrumento de medición de la Ficha de Observación, aplicada con la técnica de la Observación, utilizándose escalas cuantitativas para la variable Crecimiento Económico, medida por el PBI; y para la variable Calidad Institucional se utilizó la encuesta de IPSOS APOYO.

El objetivo de la investigación fue determinar la Influencia de la Calidad Institucional en el Crecimiento Económico del Perú. Estableciéndose la siguiente hipótesis: Si el nivel de calidad institucional es bajo, entonces en el Perú el nivel de calidad institucional influye desfavorablemente en su crecimiento económico; determinándose la influencia de la variable Calidad Institucional en el Crecimiento económico del Perú. Los resultados determinaron que en el Perú el crecimiento económico es sostenido en términos de PBI, cuyas variaciones porcentuales alcanzaron del 0,8 al 9,80\%. Asimismo se halló que la calidad institucional se encuentra en el rango de bajo; con estos resultados se comprobó que en el Perú durante el periodo 2006-2010, la baja Calidad Institucional influyó desfavorablemente en su Crecimiento Económico.
\end{abstract}

Palabras Clave: Calidad Institucional. Crecimiento Económico, PBI, Eficiencia, Integridad Institucional.

\begin{abstract}
:
Regarding the materials and methods, the study has to do with a research of documentary whose type corresponds to the relational level. The information was obtained through the application of the measuring instrument of the Observation Form, which applied the technique of observation, using quantitative scales for variable Economic Growth, measured by GDP, and Institutional Quality Ipsos Apoyo poll was used as a variable.

The objective of the research was to determine the influence of Institutional Quality in Economic Growth of Peru. Having established the following hypothesis: If the level of institutional quality is low, then in Peru institutional quality level adversely affects the economic growth, determining the influence of the variable quality of institutions in economic growth of Peru. The results determined that, in Peru the sustained economic growth in terms of GDP reached 0.8 percentage changes to $9.80 \%$. It was also found that institutional quality is in a low range; as a result we concluded that in Peru during the period 2006-2010, low institutional quality adversely influenced economic growth.
\end{abstract}

Keywords: Institutional Quality, Economic growth, GDP, Efficiency, Institutional Integrity.

\footnotetext{
' Doctor en Administración, Magister en Docencia Universitaria, Ingeniero Economista. Docente Principal de la Facultad de Ciencias Jurídicas y Empresariales de la Universidad Nacional Jorge Basadre Grohmann de Tacna.
} 


\section{INTRODUCCIÓN}

En el Perú hemos sido testigos que los diferentes sectores del Estado tienen una baja calidad institucional; la corrupción tomó él poder y se convirtió en un sistema, pero nuestro país no es el único que ha sufrido este tipo de problemas y, lo que es más, cada vez se hace más visible que la corrupción está tomando mucha mayor fuerza para convertirse en un fenómeno de naturaleza trasnacional.

Los diferentes actos y hechos de corrupción de los diferentes sectores del Estado afectan al crecimiento económico del país, a su competitividad y están estrechamente ligados a la desigualdad en el reparto de la riqueza. Los países pobres o en vías de desarrollo son los que sufren con mayor intensidad este obstáculo a la inversión.

El término "corrupción" tiene muchas aceptaciones dada su multidimensionalidad. La complejidad de definir el concepto radica especialmente en la variedad de perspectivas disciplinarias y profesionales que lo abordan. También son diversas las esferas institucionales donde se manifiesta, se analiza y se intenta controlar como problema social.

En el Perú, uno de los factores que están repercutiendo en su crecimiento económico, es sin duda la calidad de sus instituciones, ya que es frecuente escuchar lo actos de corrupción y deficiencia lo que provoca la falta de credibilidad en ellas.

Uno de los sectores que presenta menor credibilidad en su gestión es el político, ya que ha sido objeto de una serie de denuncias, que ha causado escándalo y malestar en la población. Los líderes políticos hoy en día han dejado mucho que desear por los innumerables actos de corrupción y de mal gobierno que presentan.

En cuanto al sector administrativo; se observa que su administración es lenta y deficiente; y que para que este estado se revierta más de un usuario ha sido objeto de coima por sus administradores.

La evidencia que muestran todos los estudios empíricos sobre las implicancias en diferentes variables económicas y sociales en todo el mundo. Esta problemática no es ajena al Perú; constituyendo un problema de interés social y económico para el país; por lo que motivó al estudio del presente Trabajo de Investigación denominado:"INFLUENCIA DEL NIVEL DE CALIDAD INSTITUCIONAL EN EL CRECIMIENTO ECONÓMICO DEL PERÚ/20062010."

\section{METODOLOGÍA}

\subsection{Materiales y Métodos}

El tipo de investigación es básica, de naturaleza descriptiva relacional, debido a que está basado en medir las propiedades de las variables en estudio: Calidad Institucional y Crecimiento económico.
Se adoptó el diseño (no experimental) descriptivo relacional, puesto que no se manipula la variable independiente; y se percibe medir la correlación directa existente entre dos o más conceptos o variables.

\subsection{Población y Muestra}

\subsubsection{Población}

Considerando el propósito y los objetivos de la investigación, no se consideró población y muestra de estudio.

\subsection{Técnicas e Instrumentos de Recolección de Datos}

\subsubsection{Técnicas}

La técnica para la aplicación del instrumento fue la encuesta de IPSOS APOYO; la cual fue aplicada a los pobladores mayores de 18 años. Así también se utilizó la técnica del análisis documental para el instrumento de medición de la ficha de análisis documental; aplicada para el análisis del Crecimiento Económico.

\subsubsection{Instrumentos para la Recolección de los Datos}

Para llevar a cabo los objetivos propuestos en el presente trabajo, se utilizaron como instrumento, el cuestionario; así como la ficha de análisis documental.

\subsection{Técnicas de Análisis de datos}

La información se procesó electrónicamente, utilizando para ello el programa Microsoft Excel versión Vista; y el software SPSS (versión 15) para presentar los ítems mediante gráficas y, al mismo tiempo estimar las frecuencias absolutas y relativas de cada variable.

\section{RESULTADOS}

\subsection{Crecimiento Económico Del Perú Año 2006- 2010.}

Análisis e Interpretación de Resultados:

Tabla N01: Crecimiento Económico del Perú

\begin{tabular}{|c|c|c|c|c|c|c|}
\hline \multirow[b]{2}{*}{ Ano } & \multirow{2}{*}{$\begin{array}{c}\text { INDICE } \\
\text { DE, PBA } \\
\text { (var. } \\
\text { porrentual) }\end{array}$} & \multicolumn{3}{|c|}{ Demandh interm } & \multirow[b]{2}{*}{$\begin{array}{c}\text { Exportacion } \\
\text { es }(\%)\end{array}$} & \multirow[b]{2}{*}{$\begin{array}{l}\text { Importacio nes } \\
109\end{array}$} \\
\hline & & $\begin{array}{l}\text { Cpr. } \\
(\%)\end{array}$ & $\begin{array}{l}\text { Opu } \\
0 \%\end{array}$ & $\begin{array}{l}\text { Itrit. } \\
(\%)\end{array}$ & & \\
\hline 2006 & 7,7 & 618 & 9,5 & 20,0 & 28,5 & 19,9 \\
\hline 2007 & 8,9 & 61,5 & 9,0 & 2,8 & 29,1 & 22,4 \\
\hline 2008 & 9,8 & 640 & 9,0 & 26,9 & 27,3 & 27,1 \\
\hline 2009 & Q.9 & 65,4 & 10,3 & 20,7 & 24,0 & 20,4 \\
\hline 2010 & 8,8 & 61,9 & 10,2 & 25,0 & 25,7 & 22,8 \\
\hline
\end{tabular}

Fuente: BCR. Elaboración: Propia 


\section{ÍNDICE DEL PBI}

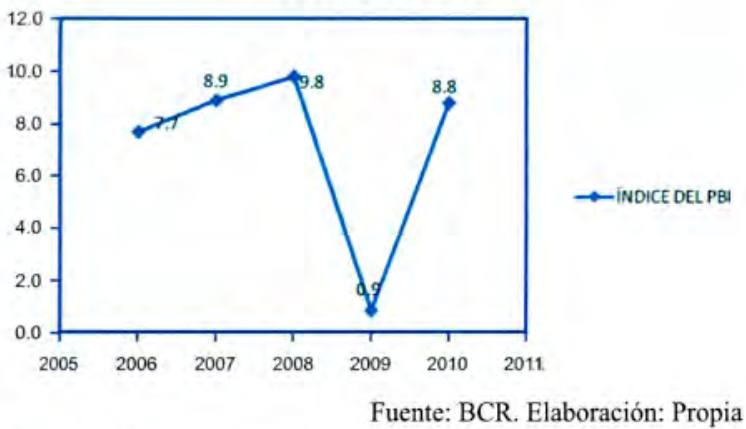

Figura $\mathrm{N}^{\circ}$ 01: Índice del PBI

En la Tabla $\mathrm{N}^{\circ} 01$ y Figura $\mathrm{N}^{\circ} 01$ anterior se pueden observar el índice del crecimiento económico del Perú para el período 2006-2010; el cual se encuentra en función de sus componentes demanda interna (consumo privado, consumo público, inversión bruta interna) exportaciones e importaciones.

Las variaciones porcentuales del índice del PBI son las siguientes; 7,7 (2006); 8,9 (2007); 9,8 (2008); 0,9 (2009); y, 8,8 para el año 2010.

Del año 2006 al 2007 se muestra un crecimiento constante cuyo incremento va en más de una unidad porcentual, llegando al índice más alto en el 2007 de $8.90 \%$ en PBI como se muestra en la Tabla (01), para el año 2008 se muestra un ligero ascenso en $0.90 \%$ según los cálculos realizados por el Ministerio de Economía y Finanzas (MEF) y que coinciden con los cálculos realizados por la Comisión Económica para América Latina y el Caribe (CEPAL), del mismo modo presenta otra disminución para el año 2009 cayendo aproximadamente a $0.90 \%$ para ese año.

Tal decrecimiento se muestra a nivel mundial, similar caída se espera para los países que tienen crecimientos importantes, China decreció en aproximadamente en $1 \%$, lo mismo ocurrirá con la India que son los países que están por encima del Perú en crecimiento económico según el Fondo Monetario Internacional (FMI).

Para el año 2010 se mostró que tuvimos un crecimiento de $8.80 \%$ en $\mathrm{PBI}$, en comparación a otros países latinoamericanos nuestro país tiene el mayor crecimiento económico además el de menor riesgo país ubicándose por debajo del mínimo, esto hace que nuestro país se haya convertido altamente atractivo para las inversiones privadas internacionales y que están interesados en invertir en el Perú, nuestra economía logró estabilizarse, nuestra moneda está más sólida en el mercado de valores, se logró mayor orden social, estamos en camino al primer mundo económicamente hablando, del mismo modo las exportaciones han crecido favorablemente en los últimos años generando beneficios importantes para la economía nacional, la producción interna también presenta incrementos esperanzadores para el futuro de país, las reservas internacionales netas alcanzarían los 30,733 millones de dólares, se abren mayores oportunidades comerciales con otros países, etc.
Se tiene conocimiento que durante el período de estudio las exportaciones totales fueron desde 7026 millones de US\$ para el año 2006; hasta 31,666 millones de dólares en el año 2010. Destacándose que los principales destinos de las exportaciones son: Estados Unidos, China, Chile, Canadá, Suiza, Japón, Holanda, España, Alemania y Brasil.

El crecimiento económico que tiene nuestro pais es uno de los más importantes a nivel mundial, es el único país latinoamericano que se encuentra dentro de los cinco crecimientos más importantes del mundo en economía, además cuya velocidad de crecimiento tiene una fuerte tendencia a estabilizarse sólidamente como una de las economías más importantes del mundo actual.

\subsection{ANÁLISIS DE LA CALIDAD \\ INSTITUCIONAL EN EL PERÚ 2006-2010}

Cuáles son los tres principales problemas del país en la actualidad?

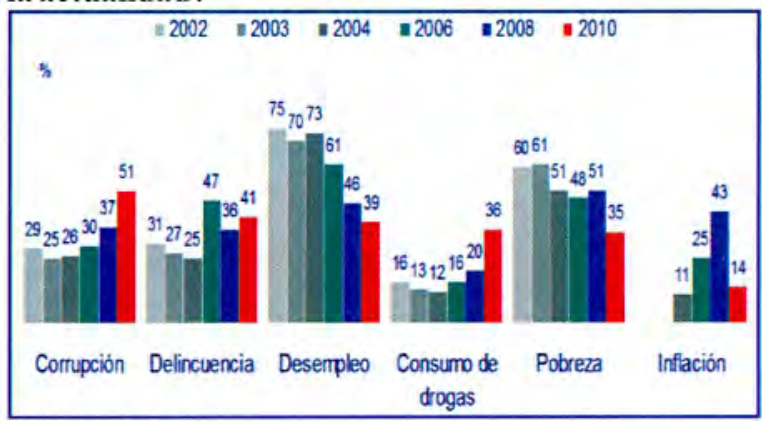

Figura $\mathrm{N}^{\circ}$ 02: Fuente: Encuesta IPSOS APOYO. Elaboración: Propia

En la Figura $\mathrm{N}^{\circ} 02$, se observa que el mayor problema del país en la actualidad es el desempleo; seguido de la pobreza; la corrupción, la delincuencia y la inflación.

Por lo que se puede concluir que el mayor problema del país durante el período 2006 al 2010 fue el desempleo; producto de la recesión económica del país.

¿Cuál le parece el principal problema que enfrenta el Estado y que le impide lograr el desarrollo del país?

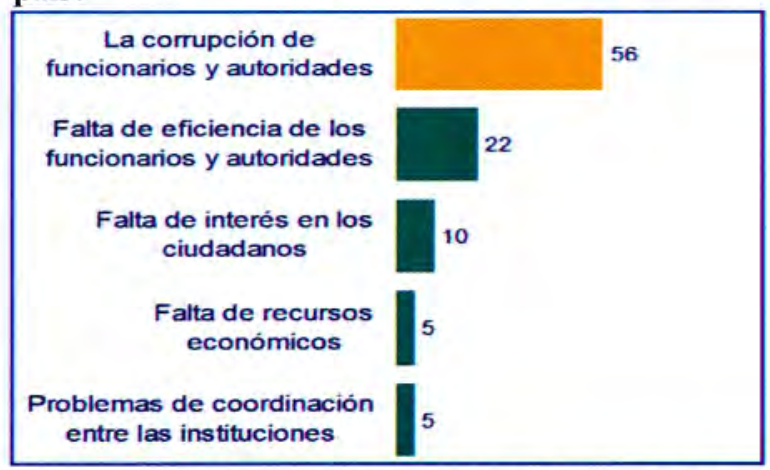

Figura $\mathrm{N}^{\circ}$ 03: Fuente: Encuesta IPSOS APOYO. Elaboración: Propia

El mayor problema que afronta el estado que le impide lograr el desarrollo del país es la corrupción de funcionarios y autoridades; el $22 \%$ de los ciudadanos señala que es la falta de efíciencia de los funcionarios y autoridades; el $10 \%$ señala que es la falta de interés de los ciudadanos; el $5 \%$ señala que es la falta de recursos económicos; y los problemas de coordinación entre las instituciones. 
En los últimos doce meses, ¿le solicitaron o dio regalos, propinas, sobornos o coimas a algún funcionario del Estado?

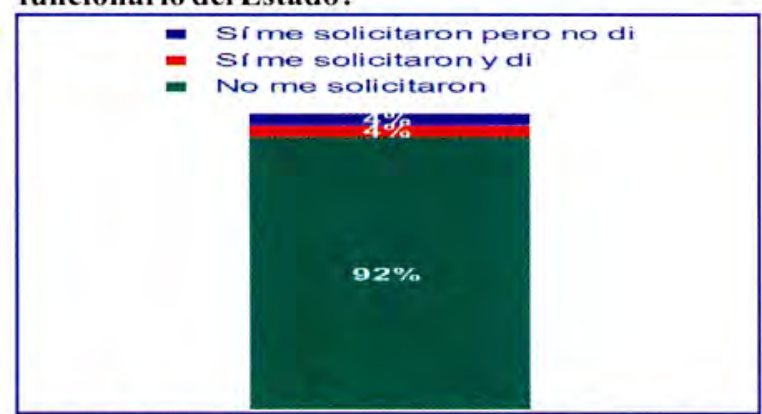

Figura $N^{\circ} 04$ : Fuente: Encuesta IPSOS APOYO. Elaboración: Propia

En la Figura $\mathrm{N}^{\circ} 04$, se observa que la mayoría de los ciudadanos encuestados señalaron que en los últimos doce meses, NO le solicitaron o dio regalos, propinas, sobornos o coimas a algún funcionario del Estado; y en menores porcentajes del $4 \%$ manifestaron que si le solicitaron y accedieron ante lo solicitado.

¿Cuán efectivos cree que son los resultados de las denuncias por casos de corrupción?

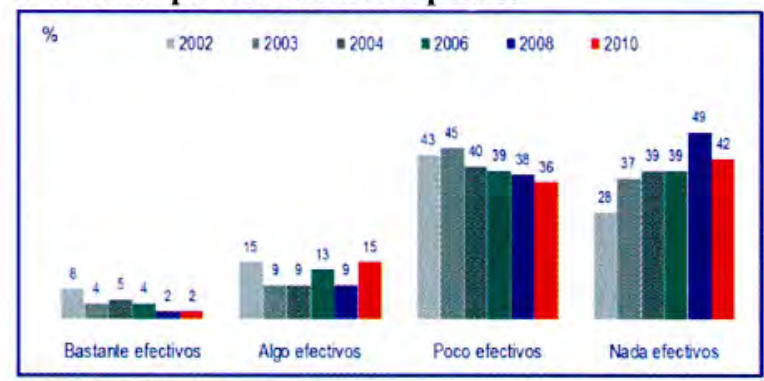

Figura $\mathbf{N}^{\circ}$ 05: Fuente: Encuesta IPSOS APOYO. Elaboración: Propia

En la Figura $\mathrm{N}^{\circ} 05$, se observa que la mayoría de los ciudadanos encuestados manifestó que los resultados de las denuncias por casos de corrupción son nada efectivos; en porcentajes menores señalan que son poco $\mathrm{y}$ algo efectivos respectivamente. Por lo que se puede concluir que las autoridades poco o nada hacer por velar por la integridad moral de las personas; permitiendo este tipo de actos delictivos en el país.

Diría usted que el Perú es un país...?.

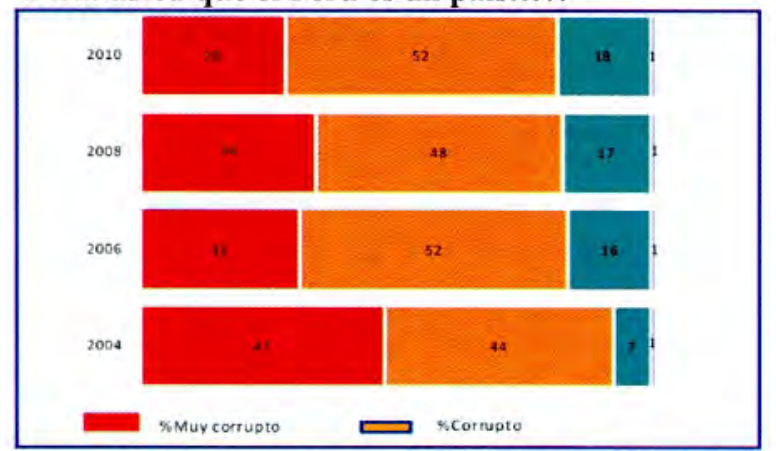

Figura $\mathbf{N}^{\circ}$ 06: Fuente: Encuesta IPSOS APOYO. Elaboración: Propia

En la Figura $\mathrm{N}^{\circ} 06$, se puede observar que el Perú es un pais corrupto; los altos porcentajes mostrados en la encuesta de IPSOS APOYO, nos evidencia estos resultados. Por lo que se puede concluir que la corrupción en el país está en aumento por falta de valores y principios de la universidad peruana.
Cuán interesado cree usted que está el gobierno actual en la lucha contra la corrupción?

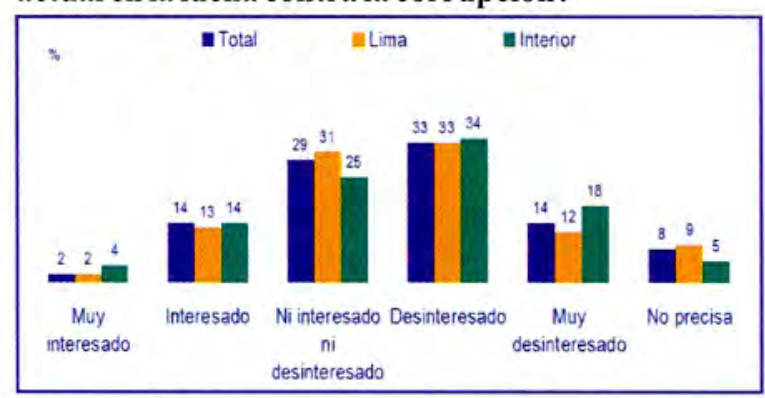

Figura $\mathrm{N}^{\circ}$ 07: Fuente: Encuesta IPSOS APOYO. Elaboración: Propia

En la Figura $\mathrm{N}^{\circ} 07$, se puede observar que la mayor parte de los encuestados manifestaron en porcentajes del $33 \mathrm{al}$ $34 \%$ que el gobierno actual no se encuentra interesado de eliminar la corrupción del país; el 25 al $31 \%$ señalan que le da lo mismo y en menores porcentajes no precisa sobre el respecto.

¿En qué gobierno considera usted que hubo más corrupción?

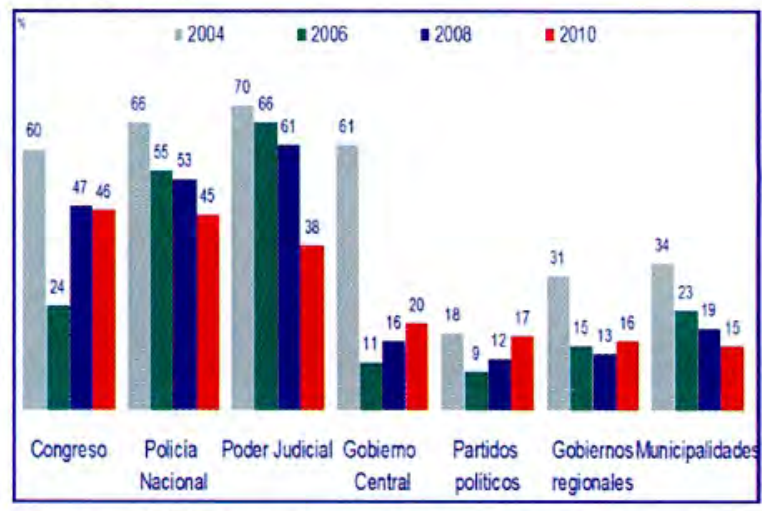

Figura $\mathbf{N}^{\circ}$ 08: Fuente: Encuesta IPSOS APOYO. Elaboración: Propia

En la Figura $\mathrm{N}^{\circ} 08$, se observa que entre los mayores porcentajes de actos corruptos se dieron lugar en el gobierno del Ing. Fujimori; seguido del primer gobierno de García; y en porcentajes del 6 al 12 manifestaron que se dio lugar en el gobierno de Toledo.

Cuáles son las instituciones más corruptas de nuestro país?.

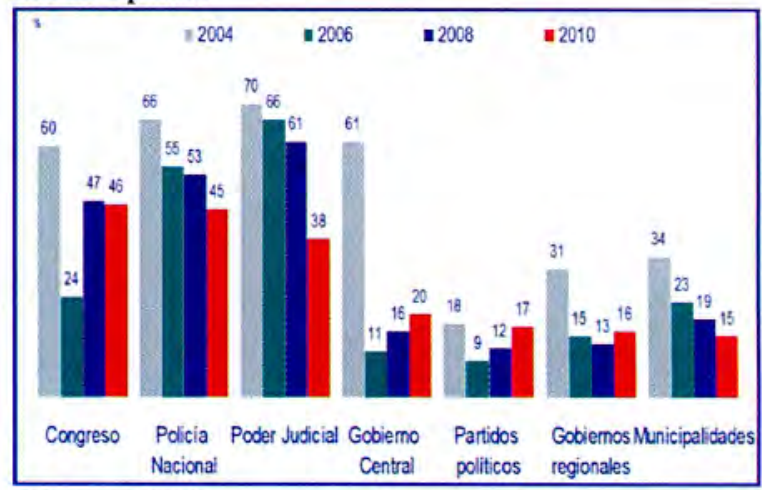

Figura N09: Fuente: Encuesta IPSOS APOYO. Elaboración: Propia

En la Figura $\mathrm{N}^{\circ} 09$, se observa que entre las instituciones más corruptas se encuentran Poder Judicial, el Congreso, Policía Nacional, el gobierno central, los gobiernos regionales y las municipalidades. 


\section{DISCUSIÓN}

1. Principales problemas del país: La pobreza, el desempleo y la inflación han dejado de ser los problemas principales y han tomado su lugar la corrupción en primer lugar $(51 \%)$ y la delincuencia en segundo $(41 \%)$. La población de nivel socioeconómico más alto parece estar más al tanto de los casos de corrupción, ya que siete de cada diez lo mencionan como el problema más grave.

3. Denuncias de casos de corrupción: La mitad de los encuestados dice no saber dónde realizar una denuncia en caso de corrupción, diez puntos porcentuales por encima del 2008. En caso de realizarse la denuncia, la mayoría de ciudadanos percibe que el trámite es poco o nada efectivo.

4. Lucha contra la corrupción: $\mathrm{Al}$ igual que en las mediciones anteriores, la gran mayoría de entrevistados percibe al Perú como un lugar donde existe corrupción; casi un tercio piensa que el país es "muy corrupto". En cuanto a las expectativas en la solución del problema, la opinión pública es poco optimista. Solo uno de cada diez, piensa que dentro de cinco años habrá una mejoría al respecto, mientras que, para la mayoría, la corrupción en el país seguirá igual o habrá aumentado en los próximos cinco años.

6. Democracia y política: La mayoría considera que la democracia es preferible a cualquiera otra forma de gobierno, sin embargo también señalan estar poco o nada satisfechos con la forma como este sistema de gobierno está funcionando en el país.

\section{CONCLUSIONES}

En el país la calidad institucional de los diferentes sectores del estado es baja; esto como producto de los elevados índices de corrupción que muestran; demostrándose el incremento de la corrupción en el Perú por factores referidos a la frecuencia del soborno en las diferentes instituciones del país.

En el Perú el crecimiento económico es sostenido. Desde el año 2006 al 2010 nuestro país presenta un continuo crecimiento en términos de $\mathrm{PBI}$, cuyas variaciones porcentuales alcanzaron del 0,8 al $9,80 \%$; producto del incremento de la producción interna de los diferentes sectores productivos del país. En condiciones de vigencia de transparencia el crecimiento debió ser mayor ya que es de conocimiento que el país ha incrementado sus actividades productivas y comerciales.

En el Perú la baja calidad institucional de los diferentes sectores del estado influye desfavorablemente en el crecimiento económico. La baja calidad de las instituciones producto de su ineficiencia y corrupción; constituyen el principal freno al crecimiento económico del Perú, a su competitividad y está estrechamente ligado a la desigualdad en el reparto de la riqueza.

La elevada tendencia en los índices de corrupción en las instituciones del estado evidencian su mala imagen institucional; las denuncias y casos sobre corrupción en el sector público afirman esta percepción.

El estado peruano poco o nada hace para aplacar este mal; en los últimos años se ha convertido en su agente promotor; siendo el partícipe en los más sonados casos de corrupción en el país.

\section{REFERENCIAS BIBLIOGRÁFICAS}

1. Busquets, Renato (2003). "Factores que propiciaron la corrupción en México. Un análisis del soborno a nivel estatal", 3er Lugar del Premio Anual de Investigación en Corrupción 2003, SFP.

2. Carbonell, Miguel y Rodolfo Vázquez comp. (2004). "Poder, Derecho y Corrupción", Siglo XXI editores, México, D.F.

3. Cermeño, Rodolfo (2001). "Decrecimiento y convergencia de los estados mexicanos", El Trimestre Económico, No. 27, pg. 603-629.

4. Glaeser, E., R. La Porta, F. López de Silanes y A. Schleifer (2004): "Do Institutions Cause Growth?", Journal of Economic Growth, 9, 3,pp. 271-303.

5. Sebastián, C. (2004): "Política macroeconómica, Instituciones y Desarrollo", en Pérez, J., Sebastián, C. y Tedde, P. Economía e Historia. Estudios en Homenaje a L. Ángel Rojo. Editorial Complutense. Madrid.

6. Soto, H. de (2000): The Mystery of Capital, Bantam Press, Random House. (Hay traducción castellana: El Misterio del Capital, Ed. El Comercio. Lima.

7. Soto, H. de (1986): El Otro Sendero, Editorial Barranco. Lima.

8. Stone, A., B. Levy y R. Paredes (1996): "Public Institutions and Private Transactions: A Comparative Analysis of the Legal an Regulatory Environment for Business Transactions in Brazil and Chile", en L. J.

\section{Correspondencia:}

Jesús Amadeo Olivera Cáceres

Urb. Universitaria A-13, Leguia. Tacna.

Ciudad Universitaria "Los Granados"

Av. Miraflores s/n. Tacna. Perú

jamadeo.olivera@gmail.com 